AperTO - Archivio Istituzionale Open Access dell'Università di Torino

Mechanical Thrombectomy in Distal Residual Occlusions of the Middle Cerebral Artery after Large Vessel Recanalization in Acute Stroke: 2b or not 2b? A Pragmatic Approach in Real-Life Scenarios

This is a pre print version of the following article:

Original Citation:

Availability:

This version is available http://hdl.handle.net/2318/1800864

since 2021-09-10T17:47:25Z

Published version:

DOI:10.1016/j.wneu.2021.04.127

Terms of use:

Open Access

Anyone can freely access the full text of works made available as "Open Access". Works made available under a Creative Commons license can be used according to the terms and conditions of said license. Use of all other works requires consent of the right holder (author or publisher) if not exempted from copyright protection by the applicable law. 


\title{
Mechanical Thrombectomy in Distal Residual Occlusions of the Middle Cerebral Artery after Large Vessel Recanalization in Acute Stroke: $2 b$ or not 2b? A Pragmatic Approach in Real-Life Scenarios
}

\author{
Riccardo Russo ${ }^{1,3}$, Bruno Del Sette ${ }^{1,4}$, Katsuhiro Mizutani $^{1}$, Oguzhan Coskun ${ }^{1}$, Federico Di Maria ${ }^{1}$, Bertrand Lapergue ${ }^{2}$, Adrien Wang $^{2}$, Mauro $^{2}$ \\ Bergui $^{3}$, Georges Rodesch $^{l}$, Arturo Consoli ${ }^{l}$
}

\begin{abstract}
- BACKGROUND /OBJECTIVE: Recent studies have suggested that a recanalization grade of modified Thrombolysis in Cerebral Infarction (mTICI) score $\$ 2 \mathrm{c}$ is strongly related with good clinical outcome rather than the current therapeutic angiography target $\$ 2 \mathrm{~b}$. To achieve better recanalization, additional further maneuvers on distal residual vessel occlusion (RVO) may be required. The aim of this study was to evaluate the safety and efficacy of rescue treatment in RVOs after recanalization of large vessel occlusions in the anterior circulation.
\end{abstract}

- METHODS: A single-center retrospective review of a prospectively maintained stroke databank was performed. Patients presenting with RVOs after mechanical thrombectomy on the M1/internal carotid artery terminus were included and further divided into treated and untreated groups: the former underwent additional maneuvers on RVOs, whereas the latter did not. Baseline and posttreatment clinical, radiologic, and angiographic data were compared between the 2 groups. End points included good functional outcome (modified Rankin Scale [mRS] score £2) rates of hemorrhagic transformations, neurologic deterio- ration and mortality.
- RESULTS: RVOs were observed in 183/488 patients (37.5\%). 74/183 (40.4\%) underwent rescue treatment, showing a better outcome in terms of median 24 hours National Institutes of Health Stroke Scale score (13 vs. 18 ;

$P<0001), 24$ hours Alberta Stroke Programme Early CT Score (6 vs. $5 ; P<0.001$ ) and 3 months mRS score $0 \mathrm{e} 2$ $(47.3 \%$ vs. $33.1 \%$; $P$ 工 0.06$)$. Recanalization of the superior

(frontal) branch of the middle cerebral artery was particularly critical in terms of outcome. Hemorrhagic transformation was higher in the untreated group $(53.6 \%$ vs. $66.6 \% ; P \llbracket 0.1)$ as well as symptomatic intracranial hemorrhage (13.1\% vs. $29.4 \%$; $P$ โ 0.01$)$. Neurologic deterioration occurred more often among untreated patients $(16.2 \%$ vs. $25.7 \% ; P\lceil 0.1)$. Three complications (1.3\%) occurred during rescue treatment.

- CONCLUSIONS: When feasible, improving mTICI score $2 a-2 b$ recanalization to mTICI $2 c / 3$ is safe and associated with a better clinical outcome, particularly for residual occlusions involving the superior branch of bifurcation.
Key words

- Distal vesselocclusions

- Ischemic stroke

- Mechanical thrombectomy

- Residual occlusions

Abbreviations and Acronyms

ASPECTS: Alberta Stroke Programme Early CT Score

CT: Computed tomography

ICA: Internal carotid artery

IV r-tPA: Intravenous recombinant tissue plasminogen activator

LVO: Large vessel occlusion

MCA: Middle cerebral artery

MT: Mechanical thrombectomy

mTICI: Modified Thrombolysis in Cerebral Infarction

mRS: Modified Rankin Scale

NIHSS: National Institutes of Health Stroke Scale
NTE: New territory embolization

RVO: Residual vessel occlusion

From the ${ }^{I}$ Department of Diagnostic and Interventional Neuroradiology and ${ }^{2}$ Stroke Center Neurology Division, Hopital Foch, Suresnes, Île-de-France, France;

${ }^{3}$ Interventional Neuroradiology, AOU Cittàdella Salute e della Scienza di Torino, Torino,

Italy; and ${ }^{4}$ Azienda Ospedaliero-Universitaria Maggiore della Carità, Novara, Italy

To whom correspondence should be addressed: Riccardo Russo,

M.D. [E-mail: russoriccardo18@gmail.com]

Citation: World Neurosurg. (2021) 151:e793-e802.

https://doi.org/10.1016/j.wneu.2021.04.127

Journal homepage: www.journals.elsevier.com/world-neurosurgery

Available online: www.sciencedirect.com

1878-8750/\$ - see front matter ${ }^{\text {a }} 2021$ Elsevier Inc. All rights reserved. 


\section{INTRODUCTION}

urrent evidence supports the concept that endovascular treatment improves outcomes in appropriately selected patients with acute ischemic stroke caused by large vessel occlusion (LVO). ${ }^{1-3}$ However, few data have been reported for distal residual vessel occlusions (RVOs), so-called downstream emboli, after the recanalization of a primary LVO. ${ }^{4}$ Although some studies have reported the need for rescue maneuvers in $23 \%$ e $44 \%$

of patients, ${ }^{5,6}$ this rate also includes the maneuvers performed on the initial LVO and not only on the RVOs. Furthermore, although mechanical thrombectomy (MT) in RVOs could improve the final recanalization grade, the risk of arterial perforation or dissection seems to be higher in distal occlusions $^{7}$ and the clinical impact

of rescue maneuvers in distal residual occlusions has not yet been investigated. We conducted a retrospective analysis concerning the management of RVOs persisting after the recanalization of M1emiddle cerebral artery (MCA) (M1 segment of MCA) and/or internal carotid artery (ICA) termination occlusion with the first-line technique. Specifically, we evaluated whether the rescue treatment used in RVOs was feasible, safe, and effective.

\section{METHODS}

Study Population

We performed a monocentric, retrospective review of prospectively collected data from our local stroke database. We reviewed the clinical files and neuroradiologic data of 702 patients treated by MT for acute ischemic stroke from January 2014 to April 2019. Posterior circulation, primary MCA-M2 isolated occlusions, and

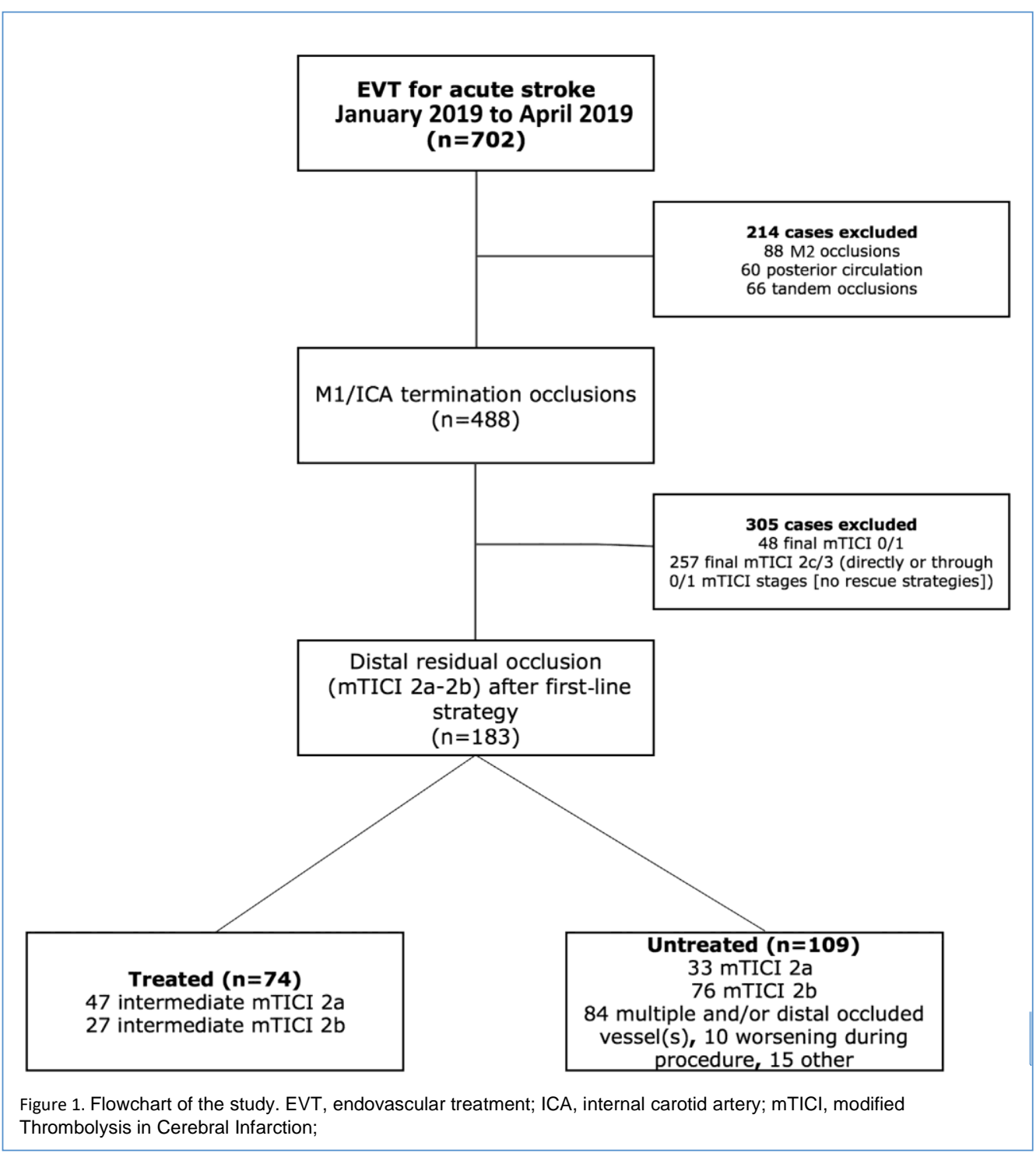




\begin{tabular}{|c|c|c|c|c|}
\hline & Treated (n โ 74) & Untreated (n โ 109) & Overall (n โ183) & $P$ \\
\hline \multicolumn{5}{|l|}{ Baseline characteristics and pretreatment data } \\
\hline Age (years) T standard deviation (range) & $66.2 \mathrm{~T} 17.1(29 \mathrm{e} 91)$ & 69.4 T14.6 (23e93) & $68.1 \mathrm{~T} 15.7(23 \mathrm{e} 93)$ & 0.08 \\
\hline Female & $31(41.9)$ & $48(44.1)$ & $79(41.7)$ & 0.8 \\
\hline Pretreatment $\mathrm{mRS}$ score $0 \mathrm{e} 2$ & $72(97.3)$ & $101(92.6)$ & $173(94.5)$ & 0.2 \\
\hline NIHSS score, median (range) & $18(0 \mathrm{e} 25)$ & $18(2 \mathrm{e} 27)$ & $18(0 \mathrm{e} 27)$ & 0.07 \\
\hline ASPECTS, median (range) & $7(1 \mathrm{e} 10)$ & $6(1 \mathrm{e} 10)$ & $7(1 \mathrm{e} 10)$ & 0.04 \\
\hline Intravenous recombinant tissue plasminogen activator & $38(51.3)$ & $51(46.8)$ & $89(48.6)$ & 0.5 \\
\hline \multicolumn{5}{|l|}{ Occlusion site } \\
\hline M1 & $57(77.1)$ & $81(74.3)$ & $138(75.5)$ & \multirow[t]{2}{*}{0.7} \\
\hline Internal carotid artery termination & $17(22.9)$ & $28(25.7)$ & $45(24.5)$ & \\
\hline Onset to groin, average (minutes) & 219.1 & 216.6 & 217.6 & 0.9 \\
\hline \multicolumn{5}{|l|}{ Treatment data } \\
\hline \multicolumn{5}{|l|}{ Collaterals } \\
\hline Poor (grade 0e2) & $33(44.6)$ & $61(55.9)$ & $94(51.36)$ & \multirow[t]{3}{*}{0.2} \\
\hline Good (grade 3e4) & $25(33.8)$ & $29(26.6)$ & $54(29.5)$ & \\
\hline Not available & $16(21.6)$ & $19(17.4)$ & $35(19.1)$ & \\
\hline Groin-to-recanalization, average (minutes) & 74.2 & 64.4 & 68.3 & 0.06 \\
\hline Onset-to-recanalization, average (minutes) & 293.1 & 281.1 & 285.9 & 0.1 \\
\hline Modified Thrombolysis in Cerebral Infarction score improvement & $64(86.4)$ & & & \\
\hline Complications & $3(1.3)^{*}$ & $5(4.6) y$ & $8(4.3)$ & 1 \\
\hline \multicolumn{5}{|l|}{ Posttreatment data } \\
\hline 24 hours NIHSS score, median (range) & $13(0 \mathrm{e} 42)$ & $18(0 \mathrm{e} 42)$ & $16(0 \mathrm{e} 42)$ & 0.001 \\
\hline 3 months mRS score $0 \mathrm{e} 2$ & $35(47.3)$ & $36(33.1)$ & $71(38.7)$ & 0.06 \\
\hline Mortality & $14(18.9)$ & $39(35.8)$ & $53(96.1)$ & 0.01 \\
\hline 24 hours ASPECTS, median (range) & $6(0 \mathrm{e} 9)$ & $5(0 \mathrm{e} 9)$ & $6(0 \mathrm{e} 9)$ & 0.001 \\
\hline Overall hemorrhagic transformation & $37 / 69(53.6) \mathrm{z}$ & $68 / 102(66.6) x$ & 105/171 (61.4)k & 0.1 \\
\hline HI1 & $8(11.6)$ & $7(6.8)$ & $15(8.7)$ & \multirow[t]{4}{*}{0.6} \\
\hline HI2 & $14(20.3)$ & $23(22.5)$ & $37(21.6)$ & \\
\hline PH1 & $10(14.5)$ & $24(23.5)$ & $34(19.8)$ & \\
\hline $\mathrm{PH} 2$ & $5(7.2)$ & $14(13.7)$ & $19(11.1)$ & \\
\hline Subarachnoid hemorrhage & $9 / 69(13.1)^{*}$ & $6 / 102(5.9) y$ & $15 / 171(8.7) \mathrm{k}$ & 0.1 \\
\hline Symptomatic intracranial hemorrhage & $9 / 69(13.1)^{*}$ & $30 / 102(29.4) y$ & $39(22.8) \mathrm{k}$ & 0.01 \\
\hline Neurologic deterioration (NIHSS $24 \leq$ NIHSS 0 p 4 ) & $12(16.2)$ & $28(25.7)$ & $40(21.8)$ & 0.1 \\
\hline \multicolumn{5}{|c|}{$\begin{array}{l}\text { mRS, modified Rankin Scale; NIHSS: National Institutes of Health Stroke Scale; ASPECTS, Alberta Stroke Programme Early CT Score; HI, hemorrhagic infarction; PH, parenchyma } \\
\text { hematoma. } \\
\text { *Including } 2 \text { new territory embolisms and } 1 \text { dissection. } \\
\text { yIncluding } 4 \text { new territory embolisms and } 1 \text { dissection. } \\
\text { z5 patients were not imaged at } 24 \text { hours. } \\
\text { x7 patients were not imaged at } 24 \text { hours. }\end{array}$} \\
\hline
\end{tabular}


tandem occlusions were excluded. Patients with final modified Treatment in Cerebral Ischemia (mTICI) scores of 0e1 and 2 ce 3 that did not require rescue treatment were also excluded. The institutional review board approved the study and waived informed consent based on the retrospective nature of the study.

Angiographic Scores and Definitions

irst-line treatment was defined as the sum of all maneuvers needed to achieve the recanalization of the LVO. We defined in- termediate mTICI as the recanalization grade achieved after all the maneuvers needed to recanalize the M1 sबgment

the ICA

ter- minus. RVOs were defined as any persistent occlusion of post- bifurcation M1-MCA branch(es) after the first-line treatment on M1/ICA. Residual isolated anterior cerebral artery occlusions were further excluded. Rescue treatment was defined as the tech-nique(s) used in case of RVOs. Final mTICI score was defined as the final angiographic result achieved after the rescue treatment. Patients with an intermediate mTICI score $2 \mathrm{ae} 2 \mathrm{~b}$ because of persistent RVOs in MCA after LVO

thrombectomy in MCA-M1 and ICA terminus occlusions were included in the study. They were divided in 2 groups: "treated" underwent further maneuvers to achieve a better

recanalization, whereas "untreated" did not

(Figure 1) according to our internal protocol.

Baseline Clinical and Neuroradiologic Data

Baseline demographics, baseline Alberta Stroke Programme Early CT Score (ASPECTS) on either magnetic resonance imaging or computed tomography (CT) scan, National Institutes of Health Stroke Scale (NIHSS) score, and intravenous recombinant tissue plasminogen activator (IV rtPA) thrombolysis administration were obtained (Table 1).

Procedural Data

A series of technical and clinical procedural and postprocedural data were collected, mainly including procedure time; onset-torecanalization time; angiographic collateral evaluation using the

American Society of Interventional and Therapeutic Neuroradiology $\operatorname{grading}^{8}$; the first-line and rescue treatment; intraprocedural complications such as perforation, dissection, new territory embolization (NTE) (i.e., embolization of the anterior cerebral

artery known to be patent before the procedure) that were considered separately for the first-line treatment and the rescue treatment; the intermediate and final mTICI scores; the type of device used; and the number of maneuvers during the first-line and the rescue treatment (Tables 1 and 2). We further evaluated the number of persistent occluded vessels at the end of the first-

line treatment (dichotomized in 1 or $>1$ and defined respectively

as single or multiple RVOs) and the type of vessel (proximal su- perior or inferior branch in cases of MCA bifurcation; specific occluded branch) (Table 2). RVO was defined as proximal whether the occlusion was located close to the origin of the M2 branch, immediately after the bifurcation, and within the area of the insular triangle in lateral projections (Figure 2). Endovascular treatment was performed under general anesthesia or conscious sedation according to the multidisciplinary evaluation, in a dedicated angiosuite with a biplane machine (Philips Clarity,

Best, The Netherlands) and by 5 experienced interventional neuroradiologists with $>9$ years' experience (range, 9e20 years). 
Two interventional neuroradiologists with 30 and 15 years' experience performed a nonblinded evaluation of all the angiograms.

Endovascular Treatment

The procedures were performed through an $8 \mathrm{~F}$ or $9 \mathrm{~F}$ sheath, with $8 \mathrm{~F}$ long introducer sheaths or balloon guiding catheters.

A wide spectrum of stentlike retrievers and aspiration catheters (alone or combined) was used; the choice of the first-line technique depended on the anatomic conditions and on the operator's judgment. All the eligible patients received IV r-tPA. According to our internal protocol, the decision whether to perform rescue

\section{Table 2. Rescue Treatment}

Rescue Treatment (N [ 74)

$\begin{array}{ll}\text { Number of occluded vessels } n(\%) & \\ 1 & 53(71.6) \\ >1 & 21(28.3)\end{array}$

Branch

Only superior (frontal/frontoparietal) division MCA or its branches

Only inferior (temporal) division MCA or its branches

Both superior and inferior branch

Both superior and inferior branch

Multiple branches occluded including anterior cerebral artery

6

Rescue technique

Stentlike retriever, $\mathrm{n}(\%)$

Contact aspiration, $\mathrm{n}(\%)$ $65(87.8)$

Combined, $n$ (\%) $6(8.1)$

Number of maneuvers, average

Periprocedural complications, $\mathrm{n}(\%)$ $3(1.3)$

Perforation, $\mathrm{n}(\%)$ 0

New territory embolization, $\mathrm{n}(\%)$ $2(2.7)$

Dissection, $\mathrm{n}(\%)$ $1(1.3)$

Final $\mathrm{mTICl}$ score $2 \mathrm{ce} 3$ 39 (52.7)

Intermediate $\mathrm{mTICl}$ score improvement, $\mathrm{n}(\%)$ $52 / 74$ (70.2)

Improved intermediate $\mathrm{mTICI}$ score with $\mathrm{mRS}$ score $0 \mathrm{e} 2$ $29 / 52$

Improvement from intermediate $\mathrm{mTICl}$ score 2a 31/47

2a improved $\mathrm{mTICl}$ score with $\mathrm{mRS}$ score $0 \mathrm{e} 2$

$15 / 31$

Improvement from intermediate $\mathrm{mTICl}$ score $2 \mathrm{~b}$

(77.7)

$2 \mathrm{~b}$ improved $\mathrm{mTICl}$ score with $\mathrm{mRS}$ score $0 \mathrm{e} 2$

$14 / 21$

MCA, middle cerebral artery; $\mathrm{mTICl}$, Modified Treatment in Cerebral Ischemia; mRS, modified Rankin Scale. 
treatment in patients with an intermediate mTICI score of 2ae2b was based on definite criteria: the distality and the number of RVOs (multiple and distal M3 occlusions were not further approached, whereas multiple and proximal ones were considered for rescue treatment), onset-to-recanalization time (patients with late recanalization obtained after several maneuvers were not further treated), the baseline magnetic resonance/CT ASPECTS

(rescue treatment was not generally performed in patients with baseline ASPECTS <4), the congruence between the ischemic area

and the cerebral tissue fed by the occluded vessel (persistent oc- clusions corresponding to brain areas without diffusionweighted imaging fluid-attenuated inversion recovery mismatch or with

early ischemic signs on CT were not considered for rescue treat- ment), age (rescue treatment was not performed in patients $>90$ years old), the baseline modified Rankin Scale (mRS) score (patients with baseline mRS score $>2$ did not undergo RS). These factors were frequently associated. At the end of the procedure, all patients were transferred to the stroke unit or to the intensive care unit.

\section{Clinical and Neuroradiologic Outcome}

The 24 hours NIHSS score was assessed by a certified neurologist, as well as 3 months mRS score, whereas the 24 hours ASPECTS was assessed by a neuroradiologist. Hemorrhagic transformation was evaluated using the ECASS (European Cooperative Acute

Stroke Study) classification. ${ }^{9}$ Symptomatic intracerebral hemorrhage, defined as hemorrhage associated with a worse neurologic status with absence of alternative explanation for deterioration, ${ }^{10}$ and neurologic deterioration (defined as $\_4$ point increase in the NIHSS score), ${ }^{11}$ were also reported. Five

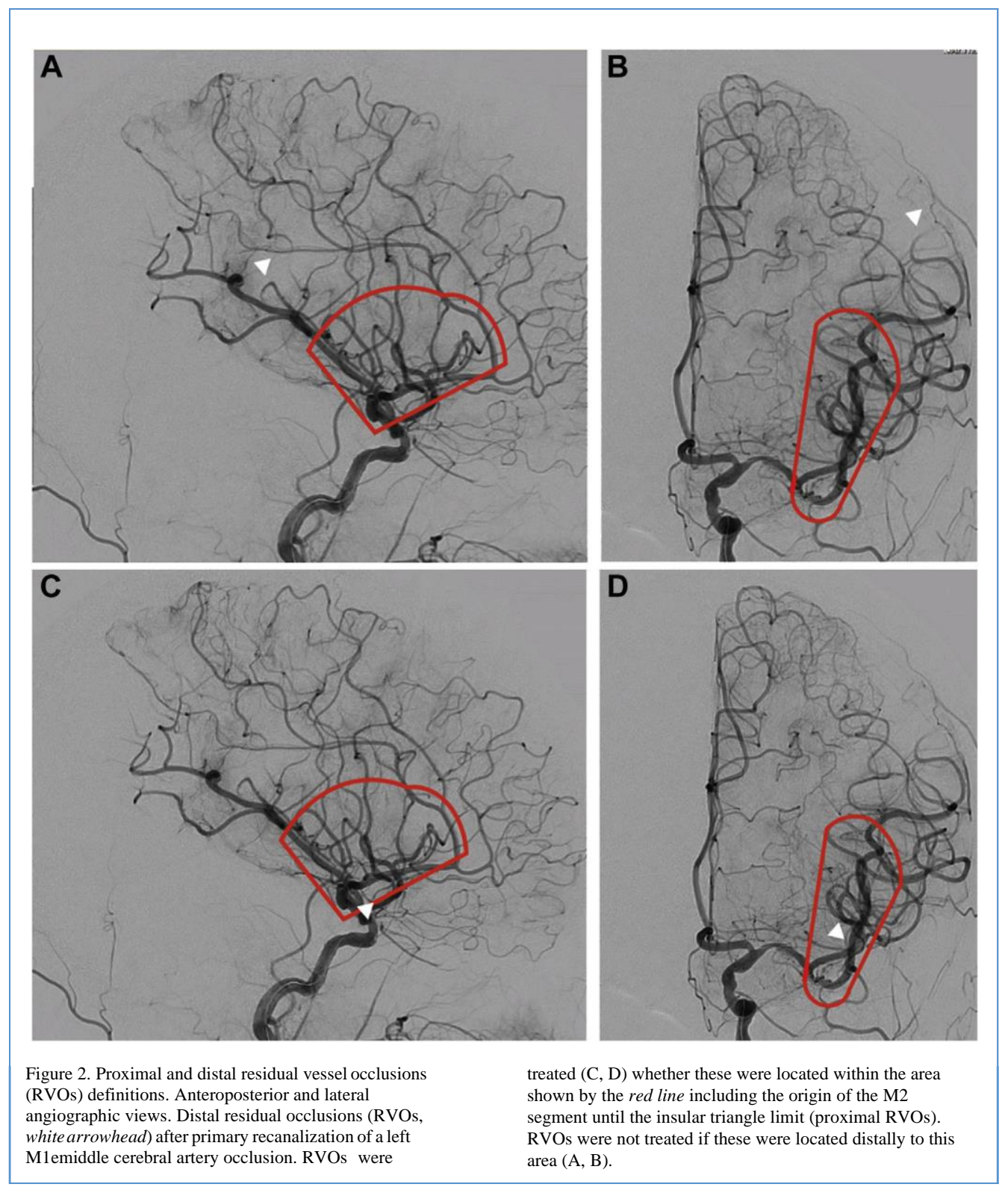




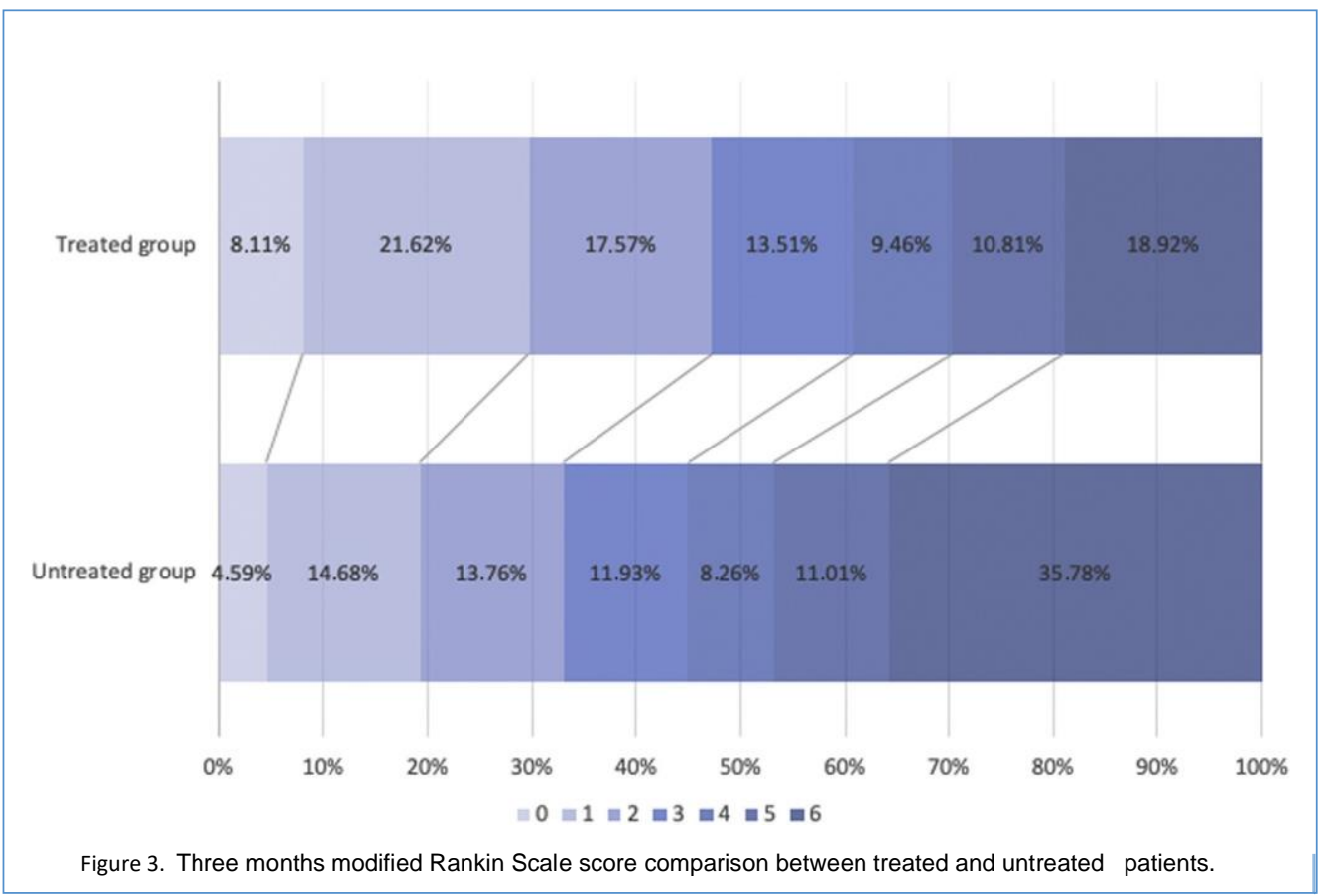

patients in the treated group and 7 patients in the untreated group were not imaged at 24 hours either because they had died or because they were immediately transferred to the referring hospital and lost to follow-up.

\section{Statistical Analysis}

A $\mathrm{c}^{2}$ test and Fisher exact test (if frequencies were <5) were used

to compare the differences of frequencies within subgroups; the Mann-Whitney test was used to compare median values and a Student $t$ test for means. A linear regression model was used to perform univariate analysis by setting 3 months mRS score, mortality, and mTICI score 2 ce 3 as determined variables. $P<$ 0.05 was considered as statistically significant. A subgroup analysis was

performed among the overall population between those patients with an intermediate mTICI score $2 \mathrm{ae} 2 \mathrm{~b}$ treated by rescue treatment and those with a final mTICI score $2 \mathrm{ae} 2 \mathrm{~b}$ who were not further treated.

Moreover, we considered that the intermediate mTICI score $2 \mathrm{~b}$ generally represents the borderline grade to consider further ma- neuvers, whereas an intermediate mTICI score $2 \mathrm{a}$ is considered an insufficient angiographic outcome and the operators could be more motivated to perform rescue treatment. Therefore, we con- ducted a secondary analysis focusing only on patients with mTICI score $2 \mathrm{~b}$ undergoing a rescue treatment (treated group) or not (untreated group) (Table 3).

We compared clinical outcomes of patients with pure RVO of the superior or the inferior branch respectively (i.e., occlusions involving only the superior or only the inferior branch of MCA bifurcation) treated by rescue treatment.

\section{RESULTS}

Baseline Characteristics

In our study, we observed RVOs after LVO MT of M1/ICA in 183/ 488 of patients (37.5\%). Among these patients, 74 (40.4\%) un- derwent rescue treatment. Demographics, clinical and radiologic features, and first-line treatment of patients undergoing rescue treatment are described in Table 1: mean age waJ 66.2 17.1 years; median NIHSS score on admission was 18 and the median ASPECTS was 7; average onset-to-recanalization time was 293,1 minutes. Rates of intermediate mTICI score $2 \mathrm{a}$ and mTICI score $2 \mathrm{~b}$ were $63.6 \%$ and $36.4 \%$, respectively. No complications occurred during the first-line treatment within the treated group, whereas 5 complications including 4 NTE and 1 dissection occurred in the untreated group during the first-line treatment.

Rescue Treatment

Of 80 patients for whom mTICI score $2 \mathrm{a}$ was achieved after first- line treatment, $47(58.7 \%)$ underwent rescue treatment, whereas for patients with intermediate mTICI score $2 b$, adjunctive ma- neuvers were performed in 27/103 (26.2\%, Figure 1). Single (71.6\%) and multiple (28.3\%) RVOs were treated. A stentlike retriever technique, direct aspiration, or a combined technique were used depending on the operator's judgment (Table 2).

Initial collateral circulation, when available, was good in 25 patients $(33.8 \%)$ and poor in $33(44.6 \%)$. Three $(1.3 \%)$ complica- tions occurred during rescue treatment, including 2 NTE and 1 dissection. NTE was associated with 3 months mRS score of 6 in both cases, whereas the dissection was associated with neurologic deterioration (3 months mRS score of 4 ).

An improvement of the mTICI score was achieved in 52 patients $(70.2 \%)$. Final mTICI score $2 \mathrm{c} / 3$ was reached in 40 patients 
Table 3. Comparison Between Patients with Intermediate

Modified Thrombolysis in Cerebral Infarction 2b Undergoing

Rescue Treatment(Treated) and Untreated Patients with Final

Modified Thrombolysis in Cerebral Infarction Score 2b After

Recanalization of the Large Vessel Occlusion

Treated Untreated

(n [27) (n โ 76)

\begin{tabular}{|c|c|c|c|}
\hline \multicolumn{4}{|l|}{ Pretreatment } \\
\hline Age (years) T standard deviation (range) & $\begin{array}{c}63.2 \\
\text { T16.4 (29 } \\
\text { e90) }\end{array}$ & $\begin{array}{c}69.4 \text { T14.6 } \\
\text { (23e93) }\end{array}$ & 0.06 \\
\hline Female & $\begin{array}{l}10 / 27 \\
(37.1)\end{array}$ & $35 / 76(46.1)$ & 0.5 \\
\hline Pretreatment $\mathrm{mRS}$ score $0 \mathrm{e} 2$ & $\begin{array}{r}27 / 27 \\
(100)\end{array}$ & $71 / 76(93.4)$ & 0.3 \\
\hline NIHSS score, median (range) & $18(0 \mathrm{e} 24)$ & $18(2 \mathrm{e} 27)$ & 0.1 \\
\hline ASPECTS, median (range) & $7(4 e 9)$ & $6(3 e 10)$ & 0.04 \\
\hline $\begin{array}{l}\text { Intravenous recombinant tissue } \\
\text { plasminogen activator }\end{array}$ & $\begin{array}{l}19 / 27 \\
(70.3)\end{array}$ & $40 / 76(52.6)$ & 0.1 \\
\hline \multicolumn{4}{|l|}{ Occlusion site } \\
\hline M1 & $\begin{array}{l}23 / 27 \\
(85.1)\end{array}$ & $56 / 76(73.7)$ & 0.2 \\
\hline Internal carotid artery termination & $\begin{array}{l}04 / 27 \\
(14.8)\end{array}$ & $20 / 76(26.3)$ & \\
\hline Onset to groin, average (minutes) & 237.6 & 212.5 & 0.3 \\
\hline
\end{tabular}

Treatment

Collaterals

Poor (grade 0e2)

Good (grade 3e4)

Not available

Groin-to-recanalization, average (minutes)

Onset-to-recanalization, average (minutes)

Modified Thrombolysis in Cerebral

Infarction improvement

Complications

Posttreatment

\begin{tabular}{|lccc|}
\hline 24 hours NIHSS score, median (range) & $11(0 \mathrm{e} 28)$ & \multicolumn{3}{c|}{16 (0e42) } & 0.02 \\
\hline mRS score 0e2 & $18(66.6)$ & $28(36.8)$ & 0.01 \\
\hline Mortality & $3(11.1)$ & $24(31.6)$ & 0.04 \\
\hline 24 hours ASPECTS, median (range) & $7(4 \mathrm{e} 9)$ & $5(0 \mathrm{e} 9)$ & 0.0003 \\
Overall hemorrhagic transformation & $13 / 26$ & $49 / 73$ & 0.1 \\
& $(50) \mathrm{y}$ & $(67.1) \mathrm{z}$ & \\
\hline HI1 & $2(7.7)$ & $4(5.5)$ & \\
\hline HI2 & $6(23.1)$ & $18(24.6)$ \\
& & \multicolumn{3}{|c|}{ Continues }
\end{tabular}

\begin{tabular}{|c|c|c|c|}
\hline & $\begin{array}{l}\text { Treated } \\
\text { ( } \mathrm{n}[27)\end{array}$ & $\begin{array}{l}\text { Untreated } \\
\text { (n [ 76) }\end{array}$ & $P$ \\
\hline PH1 & $3(11.5)$ & $19(26.1)$ & \\
\hline $\mathrm{PH} 2$ & $2(7.7)$ & $8(10.9)$ & \\
\hline Subarachnoid hemorrhage & $\begin{array}{r}3 / 26 \\
(11.5) y\end{array}$ & $5 / 73(6.8) z$ & 0.4 \\
\hline $\begin{array}{l}\text { Symptomatic intracranial hemorrhage } \\
\text { (hemorrhage NIHSS score } 24>\text { NIHSS } \\
\text { score } 0 \text { without others reasons, } n(\%)\end{array}$ & $\begin{array}{r}3 / 26 \\
(11.5) y\end{array}$ & $\begin{array}{l}20 / 73 \\
(27.4) z\end{array}$ & 0.1 \\
\hline $\begin{array}{l}\text { Neurologic deterioration (NIHSS score } 24 \\
\leq \text { NIHSS score } 0 p 4 \text { ) }\end{array}$ & $\begin{array}{l}3 / 27 \\
(11.1)\end{array}$ & $14 / 76(18.4)$ & 0.5 \\
\hline \multicolumn{4}{|c|}{$\begin{array}{l}\text { mRS, modified Rankin Scale; NIHSS, National Institutes of Health Stroke Scale; ASPECTS, } \\
\text { Alberta Stroke Programme Early CT Score; HI, hemorrhagic infarction; PH, parenchymal } \\
\text { hematoma. } \\
\text { *Including } 2 \text { new territory embolism and } 1 \text { dissection. } \\
\text { y1 patient was not imaged at } 24 \text { hours. } \\
\text { z3 patients were not imaged at } 24 \text { hours. }\end{array}$} \\
\hline
\end{tabular}

$(54,2 \%)$. The 24 hours NIHSS score and 24 hours ASPECTS me- dian were 13 and 6, respectively. Hemorrhagic transformation occurred in 37 patients (53.6\%), whereas symptomatic intracranial hemorrhage was observed in 9 $(13.1 \%)$. We observed a neurologic deterioration in 12 patients $(16.2 \%)$. Functional independence (mRS score 0e2) was achieved in 35 patients $(47.3 \%)$. Fourteen patients (18.9\%) died (Tables 1 and 2).

Comparison Between Treated and Untreated Patients with Intermediate mTICI Score $2 \mathrm{a} / 2 \mathrm{~b}$

These data were further compared with the group of patients with $2 \mathrm{a} / 2 \mathrm{~b}$ final mTICI score who did not undergo rescue treatment. The 2 groups were well matched on baseline characteristics (age, sex, basal mRS score, administration of IV r-tPA, and baseline clinical severity), although some differences were observed con- cerning age, NfiHSS score, and statistically signi cance regarding the baseline ASPECTS (Table 1).

Because the decision whether to intervene or not was taken by the interventionist with a predefined policy, later revascularization time, preprocedure lower ASPECTS, preprocedure high $\mathrm{mRS}$ score, and older age were considered in a riskebenefit assessment.

Likewise, very distal occlusions (from M3 on) were considered too dangerous and treatment was not performed.

Patients who underwent rescue treatment had higher rates of good neurologic outcome in terms of median 24 hours NIHSS score (13 vs. $18 ; P<0001)$ and 24 hours ASPECTS (6 vs. $5 ; P$ $<0.001$; Figure 3). Hemorrhagic transformation was higher, although not significantly, in the untreated group $(53.6 \%$ vs. $66.6 \% ; P 1 / 40.1$ ), whereas symptomatic intracranial hemorrhage differed significantly with a higher incidence among untreated patients (13.1\% vs. $\left.29.4 \% ; ;_{1 / 4} P \quad 0.01\right)$. Neurologic deterioration

occurred more often among untreated patients (16.2\% vs. $25.7 \% ; P \quad 1 / 40.1)$, whereas subarachnoid hemorrhage was 
Table 4. Univariate Analysis and Occluded VesselComparison

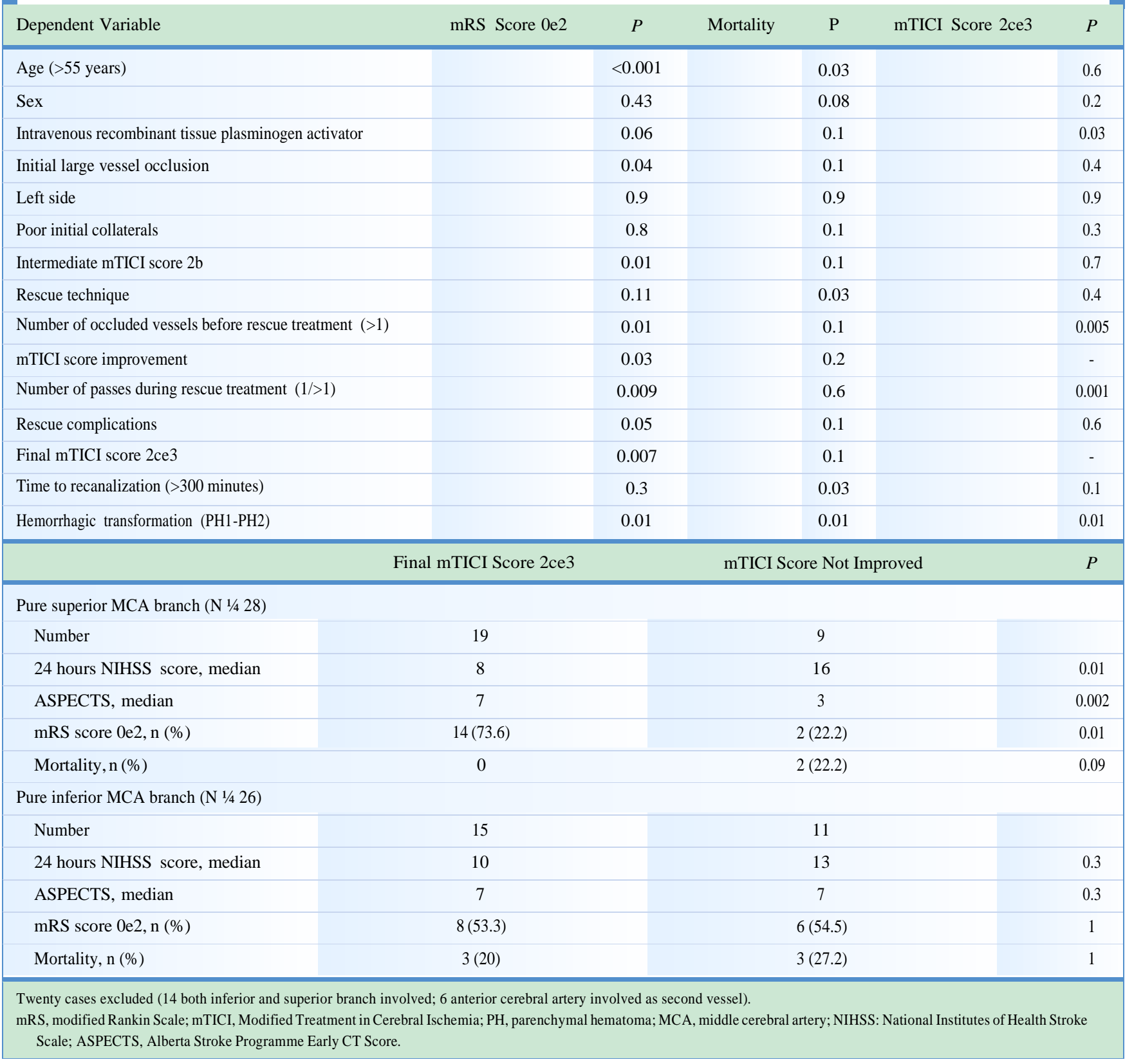

more frequent in the treated group $(13.1 \%$ vs. $5.91 \% ; P$ 0.1 ;

Table 1).

Treated patients had better clinical outcomes than did untreated patients (Table 1) and the univariate analysis (Table 4) showed that the variables that were associated with a favorable neurologic outcome ( 3 months $\mathrm{mRS}$ score $0 \mathrm{e} 2)$ were the initial M1 occlusion $/ 4$ ( $P$ 0.04), the final mTICI score $1 / 4$ ce $3(P$ $0.007)$, the mTICI score improvepnent $\left(\begin{array}{ll}P & 0.03\end{array}\right)$, the number of passes

$<2$ during rescue technique $(P 1 / 40.009)$, the parenchymal hematoma 1-2 hemorrhagic transformation $\left(P_{1 / 4} 0.01\right)$, and age $(P<0.001)$.
Mortality was higher in the untreated group (35.8\% vs. $18.9 \% ; P 0.01)$. As expected, the difference was higher in the TIEI score 2 a subgroup $(75.7 \%$ vs. $30.5 \% ; P<0.00001)$ subgroup rather that the TICI score $2 \mathrm{~b}$ subgroup (31.6\% vs. $11.1 \% ; P \quad 0.04)$.

Furthermore, we compared clinical outcomes of patients with pure occlusions of the superior or the inferior branch respectively (i.e., occlusions involving only the superior or only the inferior branch of MCA bifurcation) treated by rescue treatment. The differences observed between patients with final mTICI score 2 ce 3 after rescue treatment and those with a not improved mTICI score after rescue treatment were significant 
when the RVO was located in the superior branch rather than in the inferior one (Table 4).

TICI2b Subgroup

The results were comparable when we considered only patients with intermediate mTICI score $2 b$ undergoing rescue treatment (treated) and untreated patients with final mTICI score $2 \mathrm{~b}$ after recanalization of the LVO. Differences concerning 3 months mRS score within this subgroup were significant between treated and untreated patients $(66.6 \%$ vs. $36.8 \% ; P \quad 1 / 40.01$; Table 3).

\section{DISCUSSION}

Although the MT for acute ischemic stroke provided strong evi- dence of effectiveness, several gray areas persist. In particular, the indication to treat RVOs after a first-line treatment in LVO of the anterior circulation remains to be debated. Even if mTICI score 2 be 3 is considered the goal for $\mathrm{MT},{ }^{12}$ some investigators ${ }^{13}$ have remarked that the achievement of an mTICI score $2 \mathrm{ce} 3$ is associated with better clinical outcomes, whereas others ${ }^{14}$ have reported higher hemorrhagic complication rates in distal occlusions. Hence, although mTICI score 2 a occlusions describe a poor recanalization grade that is associated with unfavorable clinical outcome, which could justify a more aggressive approach, mTICI score $2 b$ shows a borderline condition.

Few data about the treatment of RVOs are available in the literature. A recent retrospective analysis ${ }^{15}$ including both secondarily improved mTICI score $2 \mathrm{~b}$ to mTICI score $2 \mathrm{c} / 3$ and primarily obtained mTICI score $2 \mathrm{ce} 3$ found no differences between the 2 groups in terms of outcomes and complications. Patients with intermediate mTICI score 2 a were excluded. An improvement of the recanalization grade from mTICI score $2 b$ was described in $28 / 246$ patients $(11.3 \%)$. Rescue treatment was

considered feasible and safe in some conditions and careful patient selection was emphasized. However, no clear indications were reported. To the best of our knowledge, our study represents the largest cohort providing detailed distal emboli rates on the affected vessel after MT of M1/ICA and rates of rescue treatments from mTICI score $2 \mathrm{a} / 2 \mathrm{~b}$.

Randomized clinical trials have reported rates of NTE but not the rate of distal emboli in the affected vessel. ${ }^{16}$ This situation is probably because it is difficult to distinguish whether or not the occlusion was before the endovascular treatment.

Clinical and Neuroradiologic Considerations

The management of RVOs after recanalization of the M1 segmentTin MCA ICA occlusion could be driven by the involvement of a specific vascular territory. Recently, Rosso et

al. ${ }^{17}$ retrospectively reviewed 406 patients focusing on the correlation between

different infarcted areas (based on the ASPECTS) and 3 months $\mathrm{mRS}$ score. The left internal capsule, M5, and right M3/M6 were found to be independently associated, when spared, with a good clinical outcome. In view of this finding, there may be a correlation even between the residual occluded vessel and the clinical outcome, because most of the frontal cortical territory is frequently mainly vascularized by the superior branch of the
MCA. The significant differences observed between patients with final mTICI score 2 ce 3 after rescue treatment and final mTICI

score 
$2 \mathrm{ae} 2 \mathrm{~b}$ after rescue treatment when the RVO was located in the superior branch rather than in the inferior one seem to be in line with the observations discussed earlier (Table 4). However, some confounding factors, such as the distribution of the areas of the baseline ASPECTS, should be considered.

\section{Technical Considerations}

Although both direct aspiration and stentlike retrievers have been successfully used for distal intracranial occlusions, ${ }^{18,19}$ the optimal approach remains unclear because few comparative data are available. ${ }^{7} \mathrm{We}$ compared the aspiration group (i.e., first-line aspi- ration technique) and stent-retriever/combined group (i.e.. first-line stent-retriever like/combined technique). The only difference we found concerned hemorrhagic transformation $(48.3 \%$ vs. $88.8 \% ; P 0.02)$ and mortality $(15.4 \%$ vs. $44.4 \%$; $P$ $0.05)$, which were significantly higher in the stent-retriever/combined group than in the aspiration group, although the limited sample remains a limitation factor for the interpretation of the results (Table 2).

However, these data confirm that the safety and effectiveness of rescue treatment observed in our study could be considered in line with the overall results of MT. ${ }^{20}$ Moreover, to our knowledge, no study refers to the neurologic outcome only in patients who underwent rescue treatment in RVOs after MT on M1/ICA.

Possible Indications for Rescue Treatment

As discussed earlier, the main issue remains patient selection. In our study median baseline ASPECTS (7 vs. $6 ; P$ 0.04) was the only significant difference between the treated and untreated group. This difference can be related to the decision not to treat

residual occlusions in patients with large volumes (ASPECTS

\section{$<4)$}

because of their severe prognosis, limiting the procedure to the fewest maneuvers. No other significant differences were found regarding age, pretreatment $\mathrm{mRS}$ score $0 \mathrm{e} 2$, median NIHSS score, and LVO site (Table 1). All these factors may influence the decision whether to perform an rescue treatment or not.

Therefore, even if treated patients had better clinical outcomes than did untreated patients (Table 1), a selection bias may arise from the exclusion of some patients according to our internal protocol.

The achievement of a final mTICI score 2 ce 3 was correlated to

the number of occluded vessels, the number of passes of the rescue treatment, and the IV r-tPA administration (Table 4). These data confirm that although the highest recanalization grade remains the goal for endovascular treatment, more complex procedures requiring more passes may determine longer procedures, which could result in futile recanalizations, with a potentially higher risk of hemorrhagic transformation. In these conditions, patients with multiple RVOs may be subject to a higher risk of mortality because an adequate recanalization grade would be more difficult to achieve. Furthermore, the association between the number of passes and a final mTICI score 2 ce 3 could explain that several maneuvers might not necessarily improve the recanalization grade.

As expected, we found a greater tendency to treat patients with mTICI score $2 \mathrm{a}$ compared with mTICI score $2 \mathrm{~b}$ after first-line treatment. This tendency may be explained by the fact that a potentially risky procedure such as rescue treatment in RVOs may not be justified in all situations (i.e., distal occlusions, territories with an ischemic core, noneloquent area feeding vessel, and 
multiple maneuvers required), especially if an almost optimal angiographic result (i.e., mTICI score $2 b$ ) has already been achieved, considering the risk of arterial damage, which seems to be higher in distal occlusions. ${ }^{7}$ Nevertheless, results of mTICI score

$2 \mathrm{a} 2 \mathrm{~b}$ and mTICI score $2 \mathrm{~b}$-only subgroups were comparable in terms of outcome improvement (Tables 1 and 3) Therefore, although a riskebenefit balance assessment is always mandatory, our results may encourage neurointerventionalists to try to achieve a better angiographic result in cases of an intermediate mTICI score $2 b$ when possible in selected patients, particularly when the residual occlusion involves the superior branch of bifurcation.

\section{Limitations}

This study has several limitations, in particular the retrospective (although prospectively stroke database collected data) and monocentric nature, the small sample size of subgroups (i.e., stentretriever/combined techniques group), and the selection bias that may arise from the presence of predefined criteria to perform a rescue treatment (in particular, patients with high baseline mRS score), although this represent a pragmatic, real-life approach.

\section{CONCLUSIONS}

The goal of MT is to achieve the best angiographic and subsequent clinical result. Rescue treatment, if several additional maneuvers are not required, is associated with better clinical outcomes, particularly concerning superior (frontal) branch residual occlusions. RVOs may occur frequently, and future studies should be performed to deter- mine the predictors of success of the first-line technique to achieve higher rates of firstpass effect and minimize the risk of RVOs.

\section{CRediT AUTHORSHIP CONTRIBUTION STATEMENT}

Riccardo Russo: Formal analysis, Writing - original draft. Bruno Del Sette: Writing - review \& editing. Katsuhiro Mizutani: Writing

- review \& editing. Oguzhan Coskun: Writing - review \& editing. Federico Di Maria: Writing - review \& editing. Bertrand Lapergue: Writing - review \& editing. Adrien Wang: Writing - review \& editing. Mauro Bergui: Writing - review \& editing. Georges Rodesch: Writing - review \& editing. Arturo Consoli: Methodol- ogy, Data curation, Formal analysis, Writing - original draft.

\section{REFERENCES}

1. Badhiwala JH, Nassiri F, Alhazzani W, et al Endovascular thrombectomy for acute ischemic strokeea meta-analysis. JAMA. 2015;314:18321843 .

2. Chen CJ, Ding D, Starke RM, et al. Endovascular vs medical management of acute ischemic stroke. Neurology. 2015;85:1980-1990.

3. Hong KS, Ko SB, Lee JS, et al. Endovascular recanalization therapy in acute ischemic stroke: updated meta-analysis of randomized controlled trials. J Stroke. 2015;17:268-281.

4. Settecase F. 3MAX catheter for thromboaspiration of downstream and new territory emboli after mechanical thrombectomy of large vessel occlu- sions: initial experience. Interv Neuroradiol. 2019;25: 277-2784.

5. Lapergue B, Blanc R, Gory B, et al. Effect of endovascular contact aspiration vs stent retriever on revascularization in patients with acute ischemic stroke and large vessel occlusion: the ASTER randomized clinical trial. JAMA. 2017;318: 443-452.

6. Saver JL, Jahan R, Levy EI, et al. Solitaire flow restoration device versus the Merci Retriever in patients with acute ischaemic stroke (SWIFT): a randomised, parallel-group, non-inferiority trial. Lancet. 2012;380:1241-1249.

7. Phan K, Maingard J, Kok HK, et al. Contact aspiration versus stent-retriever thrombectomy for distal middle cerebral artery occlusions in acute ischemic stroke: meta-analysis. Neurointervention. 2018;13:100-109.

8. Kim JJ, Fischbein NJ, Lu Y, et al. Regional angiographic grading system for collateral flow: correlation with cerebral infarction in patients with middle cerebral artery occlusion. Stroke. 2004;35:1340-1344.

9. Fiorelli M, Bastianello S, von Kummer R, et al. Hemorrhagic transformation within 36 hours of a cerebral infarct. Stroke. 1999;30:2280-2284.

10. Von Kummer R, Broderick JP, Campbell $\mathrm{BCV}$, et al. The Heidelberg bleeding classification: classification of bleeding events after ischemic stroke and reperfusion therapy. Stroke. 2015;46: 2981-2986.

11. Siegler JE, Martin-Schild S. Early Neurological Deterioration (END) after stroke: the END depends on the definition. Int J Stroke. 2011;6:211212.

12. Jayaraman MV, Grossberg JA, Meisel KM, et al. The clinical and radiographic importance of dis- tinguishing partial from near-complete reperfu- sion following intra-arterial stroke therapy. AJNR Am J Neuroradiol. 2013;34:135-139.

13. Dargazanli C, Consoli A, Barral M, et al. Impact of modified TICI 3 versus modified TICI $2 b$ reperfusion score to predict good outcome following endovas- cular therapy. AJNR Am J Neuroradiol. 2017;38:90-96.

14. Saber H, Narayanan S, Palla M, et al. Mechanical thrombectomy for acute ischemic stroke with occlusion of the M2 segment of the middle cerebral artery: a meta-analysis. $J$ Neurointerv Surg. 2018;10: 620-624.

15. Kaesmacher J, Maegerlein C, Zibold F, et al. Improving mTICI $2 b$ reperfusion to $\mathrm{mTICI} 2 \mathrm{c} / 3$ reperfusions: a retrospective observational study assessing technical feasibility, safety and clinical efficacy. Eur Radiol. 2018;28:274-282.

16. Andersson T, Wiesmann M, Nikoubashman O, et al. The aspirations of direct aspiration for thrombectomy in ischemic stroke: a critical anal- ysis. J Stroke. 2019;21:2-9.
Rosso C, Blanc R, Ly J, et al. Impact of infarct location on functional outcome following endovascular therapy for stroke. J Neurol Neurosurg Psy- chiatry. 2019;90:313-319.

18. Haussen DC, Eby B, Al-Bayati AR, et al. A comparative analysis of 3MAX aspiration versus 3 $\mathrm{mm}$ Trevo Retriever for distal occlusion thrombectomy in acute stroke. J Neurointerv Surg. 2020;12:279-282.

19. Altenbernd J, Kuhnt O, Hennigs S, et al. Frontline ADAPT therapy to treat patients with symptomatic M2 and M3 occlusions in acute ischemic stroke: initial experience with the Penumbra ACE and 3MAX reperfusion system. J Neurointerv Surg. 2018; 10:435-440.

20. Goyal M, Menon BK, Van Zwam WH, et al Endovascular thrombectomy after large-vessel ischaemic stroke: a meta-analysis of individual patient data from five randomised trials. Lancet. 2016;387:1723-1731.

Conflict of interest statement: The authors declare that the article content was composed in the absence of any commercial or financial relationships that could be construed as a potential conflict of interest.

Received 26 April 2021; accepted 27 April 2021

Citation: World Neurosurg. (2021) 151:e793-e802. https://doi.org/10.1016/j.wneu.2021.04.127

Journal homepage: www.journals.elsevier.com/worldneurosurgery

Available online: www.sciencedirect.com

1878-8750/\$ - see front matter ${ }^{\mathbf{a}} 2021$ Elsevier Inc. All rights reserved. 